\title{
Un programa para la enseñanza de la comprensión de ideas principales de textos expositivos
}

\author{
EDUARDO VIDAL-ABARCA \\ Universidad de Valencia

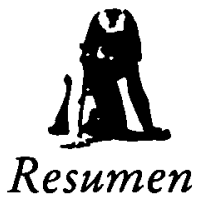

Este artículo presenta una investigación llevada a cabo con niños de $5 .^{\circ}$ curso de EGB (11 años) los cuales fueron instruidos en la captación de ideas principales de textos expositivos con estructura enumerativa y comparativa, y en la formación de la macroestructura textual. Un grupo de niños de un colegio fueron entrenados con una metodologia de instrucción directa mientras otro grupo equivalente de un centro distinto sirvió como grupo de control. Se tomaron tres tipos de medidas dependientes tras la lectura de diversos textos con un diseño pretestpostest incluyendo cada uno de ellos varias pruebas. El primer tipo de medidas se referia a la captación de ideas principales mientras el segundo y el tercero median la conciencia de estructura textual y el recuerdo respectivamente. Los resultados mostraron una ejecución significativamente mejor del grupo experimental en el postest con respecto al grupo control en la mayor parte de las medidas dependientes. Tras un periodo de seguimiento de varios meses se aplicaron de nuevo algunas pruebas manteniéndose las mejoras del grupo experimental.

Palabras clave: Comprensión lectora, Ideas principales, Instrucción directa, Macroestructura textual.

\section{A program for Teaching Comprehension of main ideas in expository texts}

\section{Abstract}

This paper reports a research study conducted with sth grade children (11 year olds), instructed in getting main ideas from expository texts with enumerative and comparative textual structures, and in forming texts' macrostructure. A direct instruction model was used to train a group of school children. Anotber group of children, equivalent in all respects, attending a different school served as control group. Three different dependent measures were obtained, after reading several texts, using a pre-postest design. The first of these measures referred to capturing main ideas from the text, while the second and third evaluated awareness of textual structure and recall, respectively. Results showed that the experimental group's performance significantly improved with respect to the control group's in the majority of dependent measures. Tests were again administered to both groups four months later, results showed that improvements were maintained.

Keywords: Reading comprehension, Main ideas, Direct instruction, Textual macrostructure.

Dirección del autor: Dpto. de Psicología Evolutiva y de la Educación. Universidad de Valencia. EU de F. de Profesorado. c/ Alcalde Reig, 8. 46006 Valencia.

Original recibido: Octubre 1989. Revisión recibida: Noviembre 1989. Aceptado: Diciembre 1989. 


\section{INTRODUCCION}

Cualquier intervención educativa en comprensión lectora, particularmente si se refiere a textos expositivos, reserva un capítulo importante para la comprensión de ideas principales lo cual no es extraño ya que esta habilidad está en la base de los macroprocesos de comprensión. El problema es que este tipo de intervención con frecuencia ha tenido y sigue teniendo un carácter ateórico, es decir, no se ha basado en una teoría acerca del procesamiento de textos escritos (Campione y Armbruster, 1985). Así, el procedimiento más habitual ha consistido en presentar diversos textos a los ninos pidiéndoles a continuación que señalaran o produjeran la idea principal de los mismos. Ello ha ocasionado que bajo el epígrafe de captación de ideas principales sea posible encontrar procesos cognitivos muy diferentes (Kieras, 1985). Así por ejemplo, se pueden hallar resultados contradictorios acerca de a qué edades son capaces los niños de producir la idea principal de textos expositivos de nivel de dificultad lectora similar (Yussen y col., 1982).

Un modelo capaz de proporcionar la base teórica a la que nos estamos refiriendo es el de Kintsch y van Dijk (Kintsch y van Dijk, 1978; van Dijk y Kintsch, 1983) el cual considera que la meta última de la comprensión es la formación de la macroestructura textual entendiendo por tal una descripción semántica abstracta del contenido del texto en orden a posibilitar una coherencia global del discurso. La construcción de esta macroestructura textual, se realizaría según este modelo, a partir de la aplicación de unas macrorreglas (supresión, generalización y construcción) a las proposiciones individuales del texto, lo cual conduciría a la formación de macroproposiciones de diferente nivel. Además de estas macrorreglas existirían unas macroestrategias que operarían sobre el conjunto del discurso. Estas macroestrategias constituirian el componente arriba-abajo (top-down) del proceso interactivo que ocurre en la comprensión y permitirían al lector hacer inferencias y anticipaciones acerca de las macroproposiciones probables del texto. El modelo distingue entre macroestrategias contextuales derivadas de los esquemas de conocimiento del lector y macroestrategias textuales dentro de las cuales van Dijk y Kintsch citan a las señales estructurales, las estrategias sintácticas del escritor, los marcadores textuales, las estrategias semánticas y las estrategias esquemáticas. La aplicación de estas macrorreglas y macroestrategias se vería facilitada por unos patrones organizativos de naturaleza esquemática consistentes en categorías convencionales que proporcionan una estructura al texto considerado como un todo. Estos patrones organizativos textuales o superestructuras, también llamados estructuras esquemáticas, han sido ampliamente estudiados en el discurso narrativo y más recientemente en el campo de la prosa expositiva. El funcionamiento de estas superestructuras en la comprensión tiene un carácter interactivo, al igual que en el caso de la macroestructura. Así, en la medida en que el texto tenga una estructura textual adecuada a los propósitos del escritor y se acompañe de señales superficiales que hagan patente dicha estructura se facilitarán los procesos abajo-arriba del lector. Igualmente, en la medida en que esas estructuras estén en la mente del lector y no sólo en el texto, se facilitarán los procesos arriba-abajo.

A pesar de que la formación de la macroestructura textual es algo que 
los lectores, al menos los más maduros, realizan ordinariamente durante la lectura, independientemente de que se les pida expresamente o de que la misma esté explícitamente afirmada en el texto, van Dijk y Kintsch constatan que en ocasiones los sujetos pueden fallar en estas macrooperaciones. Esto es particularmente evidente cuando se trata de lectores inmaduros o jóvenes, lo cual justifica una intervención específica al respecto.

Hay dos grandes tipos de variables que, siguiendo el modelo expuesto, pueden afectar a la formación de la macroestructura textual y, por tanto, a la captación de ideas principales de un pasaje expositivo: variables relativas al texto leído y variables que afectan al sujeto lector. Dentro de las primeras nos parece interesante aludir a dos subgrupos. El primero de ellos se refiere a la estructura organizativa de los textos distinguiendo diversos factores. El primero se refiere al grado de organización de los textos, concluyendo que la comprensión y el recuerdo se facilitan cuando el texto está bien organizado (Danner, 1976; Kintsch y Yarbrough, 1982; Brooks y Dansereau, 1983). El segundo factor es el tipo de estructura textual o estructura esquemática. La consideración de este factor es problemática ya que no existe una clasificación unánimemente aceptada sobre las estructuras de la prosa expositiva, siendo una de las más citadas la de Meyer $(1975,1985)$ : compilación (con las modalidades de secuencia y enumeración), causación, comparación/contraste, problema/solución y descripción. A pesar de esta dificultad, se puede decir que hay estructuras textuales (causación y comparación/contraste) que proporcionan un mejor recuerdo que otras (compilación o enumeración) (Meyer y Freedle, 1984; Richgels y col., 1987), debido posiblemente al grado de interconexión de ideas que producen en la memoria, aunque hay datos distintos cuando se toman medidas de comprensión en lugar de medidas de recuerdo (Englert y Hiebert, 1984; Englert y Thomas, 1987). El tercer factor se refiere a la existencia de una frase tópica en el texto, es decir, de una frase de la que se pueda afirmar que expresa la idea principal del pasaje, facilitando la comprensión la existencia de dicha frase (Williams, Taylor y Ganger, 1981; Williams, 1984; Hare, Rabinowitz y Schnieble, 1989). Un cuarto factor es el número de proposiciones que apoyan la idea principal del texto, el cual es igualmente un elemento facilitador (Palmere y col., 1983).

El segundo grupo de factores que pueden afectar a la formación de la macroestructura textual se refiere a diversos aspectos de la estructura superficial entre los que se citan: a) la existencia de señales textuales tales como afirmaciones explícitas acerca de la estructura de las relaciones entre las proposiciones del texto, afirmaciones que anuncian el contenido del texto, frases de resumen, señales evaluativas o títulos y encabezamientos entre otras (Meyer, Brandt, Bluth, 1980; Britton y col., 1982; Glover y col., 1988; Ohlhausen, Roller, 1988), encontrándose en general que estas señales no afectan o lo hacen de forma muy limitada a los lectores maduros, pero tienen mayor efecto en el caso de lectores inmaduros o jóvenes; $b$ ) la posición de la idea principal, concluyendo que cuando dicha posición es la inicial, se facilita la formación de la macroestructura textual (Kieras, 1979, 1981, 1982; Garner y col., 1986; Hare, Rabinowitz y Schnieble, 1989).

Dentro de las variables que afectan al sujeto lector se ha estudiado ampliamente el papel de los esquemas de conocimiento del sujeto. Un tipo de esquemas es el que se refiere al conocimieto de los sujetos sobre el domi- 
nio específico o tópico del discurso, encontrándose que dicho esquema facilita considerablemente la formación de la macroestructura textual tanto en tareas de recuerdo como de comprensión (Chiesi, Spilich y Voss, 1979; Pearson, Hansen y Gordon, 1979; Recht y Leslie, 1988). Otro tipo de esquemas de conocimiento que tiene un interés particular en esta investigación es el referido a las estructuras textuales. Centrándonos en las estructuras de la prosa expositiva, se ha encontrado que la conciencia de dichos esquemas tiene un componente claramente evolutivo de forma que los niños de grados escolares medios manifiestan menor conciencia de ellos que los muchachos de grados superiores de la escolaridad (Richgels y col., 1987; Englert y Hiebert, 1984; Englert y Thomas, 1987; Garner y col., 1986), hallándose incluso que los estudiantes universitarios son poco conscientes de esos esquemas textuales (Brooks y Dansereau, 1983; Cook y Mayer, 1988). Esta conciencia de estructura textual permite a los sujetos emplear la «estrategia estructural» (Meyer, Brandt y Bluth, 1980; Meyer, 1985; Taylor y Samuels, 1983) la cual consiste en la búsqueda y seguimiento de la estructura supraordinada del texto, centrándose en el mensaje que se intenta transmitir, de forma que se relacionen adecuadamente los detalles más importantes con dicha estructura, facilitándose asi la codificación y el recuerdo de la información mediante ese marco organizativo. Aquellos lectores inmaduros que no son capaces de emplear dicha estrategia, emplean otra claramente ineficaz para la formación de la macroestructura textual. Esta estrategia ineficaz ha sido calificada como "estrategia de listado" (default list strategy) (Meyer, 1985, 1987), o tópico-más-detalles (Scardamalia y Bereiter, 1984) y se caracteriza por no ser sistemática, no contener un plan de procesamiento del texto y ceñirse a un procesamiento superficial del mismo. En consecuencia el lector no establece un auténtico diálogo con el escritor, produciéndose una comprensión y un recuerdo de unidades de información separadas, sin otro vínculo de unión que el hecho de referirse a un determinado tópico temático.

El marco teórico que acabamos de exponer proporciona indicaciones útiles para diseñar programas instruccionales que favorezcan las estrategias de captación de las ideas principales a partir de la formación de la macroestructura textual. Así, Baumann (1984) utilizó un procedimiento de instrucción directa con niños de $6 .^{\circ}$ curso para enseñarles diversas habilidades relacionadas con la captación de ideas principales. El procedimiento empleado se mostró eficaz aunque este autor se ciñó a textos expositivos de estructura compilativa o enumerativa. Stevens (1988) realizó otro estudio con alumnos de aulas de apoyo de los cursos $6 .^{\circ} \mathrm{al} 11 .^{\circ}$ mediante enseñanza asistida por ordenador. Este autor incluyó párrafos con diferente estructura textual expositiva (descriptiva, comparativa, secuencial y causal) y comparó la efiacia de procedimientos instruccionales estratégicos con otros basados en habilidades de clasificación, encontrando que los primeros fueron claramente más efectivos que los segundos.

Junto a estos estudios directamente referidos a la enseñanza de la captación de ideas principales, existen otros que han intentado instruir a sujetos de diversas edades en el empleo de la estrategia estructural como vía de mejora de los macroprocesos lectores. Así, Brooks y Dansereau (1983) en una investigación con estudiantes universitarios lograron que los sujetos experimentales mejoraran el recuerdo de la información más importante de 
textos científicos mediante un programa de enseñanza acerca de la estructura organizativa de dichos textos, no detectándose diferencias significativas en el recuerdo de detalles. Cook y Mayer (1988) trabajando igualmente con estudiantes universitarios diseñaron un programa instruccional para mejorar la comprensión de textos expositivos con estructura de enumeración, secuencia y generalización consiguiendo incrementar el rendimiento de los sujetos experimentales en el recuerdo de la información más importante y en la resolución de cuestiones de aplicación, no encontrando tampoco diferencias significativas en la contestación a preguntas de detalle. Finalmente, Armbruster, Anderson y Ostertag (1987) instruyeron a niños de $5 .^{\circ}$ curso en la comprensión de textos expositivos con la estructura proble$\mathrm{ma} / \mathrm{solución} \mathrm{encontrando} \mathrm{que} \mathrm{el} \mathrm{procedimiento} \mathrm{de} \mathrm{enseñanza} \mathrm{aplicado} \mathrm{me-}$ joró el recuerdo de las ideas más importantes de textos con dicha estructura mientras que no se encontraron diferencias significativas entre los grupos experimental y control en la contestación a preguntas de detalle.

Siguiendo el modelo anteriormente expuesto y de acuerdo con los resultados mencionados, nuestro trabajo ha puesto a prueba la eficacia de un programa de instrucción para mejorar la capacidad de captar las ideas principales de textos expositivos con niños de grados intermedios. Pensamos que esta mejora ocurriría en la medida en que se incrementara la sensibilidad de los sujetos hacia los aspectos más importantes del mensaje escrito (Winograd, 1984) para lo cual enseñamos a los niños a utilizar la estrategia estructural. Así, nuestro estudio ha pretendido conectar las investigaciones instruccionales sobre captación de ideas principales y aquellas otras directamente enfocadas a la enseñanza de superestructuras textuales. En concreto, nosotros esperábamos en primer lugar que nuestra intervención mejorara la captación de la idea principal tanto explícita como implícita de textos expositivos adecuados al nivel escolar de los niños y que dicha mejoría se mantuviera durante un período de seguimiento. En segundo lugar esperábamos que el programa de instrucción incrementara la habilidad de jerarquizar las diferentes ideas del texto. En tercer lugar, nuestra intervención debería producir una mayor conciencia de los niños acerca de las estructuras de la prosa expositiva incluidas en el programa.

Una de las cuestiones más debatidas en cualquier programa de instrucción es la generalización de los efectos beneficiosos de la intervención a otras habilidades relacionadas. Si no existiera tal generalización, la enseñanza debería ser tan específica, que la relación coste-beneficio pondría en cuestión la misma viabilidad de muchos programas. De ahí que una de las recomendaciones más usuales es instruir estrategias generales que tengan una capacidad de generalización amplia (Pressley y col., 1989). En nuestro caso, el programa incidía únicamente en tareas de comprensión, pero dado que las estrategias instruidas eran de tipo general pensamos que los efectos beneficiosos deberían extenderse a tareas de recuerdo, ya que ambas tareas tienen componentes comunes y son complementarias en situaciones educativas. Por ello pusimos a prueba una cuarta hipótesis, pronosticando que el programa aplicado mejoraría la capacidad de recuerdo de ideas más importantes de textos expositivos, pero no el recuerdo de las las ideas menos importantes o detalles. 


\section{METODO}

\section{Sujetos y diseño}

Nuestra investigación se realizó durante el año 1988 en dos aulas de $5 .^{\circ}$ de EGB de dos Colegios de Valencia, en una de las cuales se aplicó el programa de instrucción (grupo experimental) sirviendo la otra de grupo de control. Cada aula constaba de 32 alumnos de ambos sexos. Dado que la asignación de los sujetos a los grupos experimental y control no fue aleatoria se aplicaron diversos subtets del Test de Análisis de Lecto-Escritura (TALE, subtests de lectura de palabras y de texto), así como de la Batería de Aptitudes Diferenciales y Generales (BADYG, forma E, subtests de habilidad mental verbal, comprensión verbal, habilidad mental no verbal y razonamiento lógico) no encontrándose diferencias significativas entre ambos grupos en ninguna de las pruebas.

Se empleó un diseño $2 \times 2$ con un factor entresujetos: tratamiento, (grupo experimental y grupo de control) y un factor intrasujetos: tiempo, con medidas repetidas (pretest, postest). Nuestro diseño incluyó un estudio de seguimiento cuatro meses después de finalizado el postest mediante una segunda aplicación de algunas de las medidas dependientes. Dado que los niños ya habían pasado de curso, y que algunos no habían aprobado el anterior, perdimos algunos sujetos quedando 24 en el grupo experimental y 27 en el de control.

\section{Material y procedimiento}

Para el programa de instrucción, elaboramos 26 textos de longitud aproximada entre 100 y 150 palabras, compuestos todos ellos de varios párrafos, y siendo en todos los casos adaptaciones de los libros de texto escolares de los cursos $4 .^{\circ}$ y $5 .^{\circ}$ de EGB. En dicha elaboración tuvimos en cuenta las variables textuales a las que aludimos en la introducción de este escrito. Todos los textos tenían una estructura enumerativa o comparativa de acuerdo con la reformulación que hicieron Englert y Hiebert (1984) a partir de la clasificación de Meyer. La elección de estas estructuras se justifica tanto por la mayor accesibilidad de las mismas por parte de los niños de grados escolares medios como por su mayor frecuencia en los libros de texto del área de experiencias de estos niveles. Un grupo de profesores en ejercicio juzgó la adecuación de la organización estructural de los textos y el resto de variables textuales enumeradas en la introducción.

Se empleó un procedimiento de instrucción directa (Duffy y Roehler, 1982; Pressley y col., 1989) en el que se prestaba especial atención a la enseñanza de la estrategia estructural como un medio de distinguir las ideas textuales más y menos importantes (Vidal-Abarca, 1989). El programa fue aplicado en el grupo de tratamiento por el autor de esta investigación durante el horario escolar a lo largo de 12 sesiones de 45 minutos de duración aproximadamente, distribuidas durante los meses de abril y mayo de 1988. Durante el mes de octubre el mismo grupo recibió dos sesiones de recuerdo previas al estudio de seguimiento.

El contenido, la secuencia de actividades y los procedimientos instruccionales incluídos en el programa fueron los siguientes. Durante la primera sesión el instructor modeló un procedimiento para identificar la idea prin- 
cipal explícita de un texto con estructura comparativa, relacionando dicha idea con los detalles que la apoyaban. Para ello se dividió el texto en párrafos, se buscó la información esencial de cada uno de ellos y se obtuvo posteriormente la idea principal explícita de todo el texto, entendiendo por tal aquella oración que recogía la idea de más alto nivel que abarcaba a todos los párrafos, diferenciando entre el tema del texto («de lo que el texto trata») de la idea principal ("lo que dice sobre ese tema"). A continuación el instructor modeló un procedimiento para representar la macroestructura textual. Este procedimiento utilizó una imagen gráfica, una balanza en el caso de los textos comparativos, y consistió en completar dicha imagen gráfica con las diversas macro y microproposiciones del texto (Ver Apéndice). De esta forma los niños percibían más claramente los diferentes niveles de importancia de las distintas proposiciones del texto. Todo ello fue practicado por los niños durante la segunda sesión, con un texto similar y con la guía del instructor, mediante un diálogo colectivo en el que se orientaban los procesos mentales y se solicitaba de los niños que dieran e intercambiaran razones sobre sus respuestas. Durante la tercera sesión se hizo lo mismo que en la primera, pero en este caso la idea principal estaba implícita por lo cual era necesario «inventar» la frase que podía haber escrito el autor para comunicar más claramente su propósito informativo, haciendo hincapié en que una misma idea podía ser expresada por distintas frases equivalentes. Asimismo se hizo caer en la cuenta a los niños de las señales textuales superficiales (en este caso eran expresiones de carácter comparativo) que orientaban la producción de esa idea principal. De forma semejante a como hicimos en la segunda sesión, en la cuarta los niños tuvieron una práctica guiada de lo que el instructor había modelado en la sesión precedente. Durante la quinta sesión, el instructor modeló, y a continuación los niños practicaron, la producción de un texto con estructura comparativa, siguiendo las recomendaciones de diversos autores acerca de la unión de actividades de comprensión y producción de textos en orden a favorecer las macrooperaciones de formación de la macroestructura textual (van Dijk y Kintsch, 1983; Taylor y Beach, 1984; Rubin y Hansen, 1986). La producción de un texto de estas características implicaba varios pasos: dibujar el esquema de la balanza y rellenarlo pensando primeramente qué es lo que se iba a comparar (p. e. astros luminosos y no luminosos) y con arreglo a qué categorías (p. e. origen de su luz, distancia que los separa, etc.), escribir la idea principal en un lugar aparte, buscar expresiones comparativas y anotarlas, y por fin, escribir el texto con arreglo al esquema elaborado pensando el lugar dónde se iba a incluir la idea principal y aquellas expresiones comparativas que se habían de emplear. La sexta sesión fue un resumen de las cuatro primeras y en ella los niños practicaron, de forma más independiente y con la adecuada supervisión del instructor, las habilidades aprendidas.

El contenido y secuencia de actividades de las sesiones séptima a novena fueron muy similares a los de las sesiones primera a quinta, pero empleando textos con estructura enumerativa y de una forma más abreviada, sin parcelar el texto en párrafos, ya que los niños habían aprendido el procedimiento general. Así en la sesión séptima, el instructor leía un texto con la idea principal explícita y modelaba cómo formular hipótesis desde el comienzo, acerca de la idea principal probable del texto, sometiendo dichas 
hipótesis al juicio de los niños. Posteriormente se modelaban dos actividades adicionales: diferenciar frases que pudieran ser detalles de la idea principal del texto eligiendo entre varias oraciones posibles, y puntuar diversas frases del texto según fueran muy importantes, bastante importantes y poco importantes. A continuación se modelaba el procedimiento para representar la macroestructura textual utilizando en este caso la imagen gráfica de un árbol (Ver Apéndice). En la misma sesión los niños practicaban estos mismos procesos con la guía del instructor y con un texto de similares características. La sesión octava fue idéntica a la anterior con la salvedad de que los textos tenían la idea principal implícita, lo cual se advertía previamente a los niños, y por tanto había que «inventar" la frase que podía haber escrito el autor para facilitar la comprensión, insistiendo en que una misma idea podía ser expresada con frases diferentes. La sesión novena fue similar a la quinta, es decir, se trataba de modelar la producción de un texto, con estructura enumerativa en este caso, y de guiar la producción de otro similar por parte de los niños, siguiendo unos pasos similares a los explicados en la descripción de la sesión quinta.

Desde la sesión décima a la duodécima, se practicó lo que los niños habían aprendido anteriormente además de enseñarles a distinguir las estructuras textuales comparativas de las enumerativas. Así en la sesión décima, los niños tras leer tres textos con la idea principal explícita y representativos de alguna de las estructuras textuales citadas, emparejaban cada texto con la estructura correspondiente. A continuación practicaban la representación de la macroestructura textual, mediante la imagen gráfica de la balanza o el árbol, según el tipo de estructura textual, para pasar posteriormente a la localización de la idea principal, y la diferenciación entre ideas más y menos importantes. La sesión undécima era similar a la anterior con dos salvedades: la idea principal de algunos textos estaba implícita, por lo cual los niños tenían que discriminar en cada texto si la idea principal estaba presente o no antes de escribirla, y en algunos textos los niños no tenían que rellenar la imagen gráfica de la balanza o el árbol, sino imaginarse dicha imagen ya rellenada. Es decir, se trataba de entrenar a los niños en la formación de una representación mental de la macroestructura del texto. En la sesión duodécima los niños practicaron de forma autónoma las actividades de la sesión precedente poniendo en común el resultado. Las dos sesiones del período de seguimiento fueron muy similares a estas últimas. Como se puede apreciar, a lo largo de todo el período de instrucción prestamos atención especial a las estrategias metacognitivas haciendo que los niños reflexionaran sobre las razones de sus respuestas, evaluaran la corrección de las mismas y emprendieran las medidas correctivas necesarias, todo ello en un continuo diálogo (Baker y Brown, 1984).

\section{Medidas dependientes}

Se tomaron tres tipos de medidas dependientes. El primer tipo se refería a la obtención de ideas principales de un texto. Constaba a su vez de tres pruebas: a) escribir la idea principal explícita de cuatro textos, b) escribir una frase que indicara la idea principal implícita de dos textos, c) puntuar cinco oraciones de cada uno de los dos textos de la prueba según su importancia dentro de la estructura textual $(2,1$, ó 0 de mayor a menor im- 
portancia). Todos los textos empleados eran similares a los empleados para el programa de instrucción. Se elaboraron tres pruebas equivalentes de las dos primeras medidas aplicadas, antes de comenzar el programa (pretest), inmediatamente finalizado éste (primer postest) y tras las sesiones de seguimiento (segundo postest) obteniéndose un coeficiente de consistencia interna (Nunnally, 1973) de 0.60 en la primera medida y 0.82 en la segunda. La tercera medida fue aplicada únicamente en el pretest y primer postest siendo el coeficiente alfa de 0.72 .

La corrección de la primera de las pruebas se realizó otorgando un punto por cada respuesta correcta (escritura de la idea principal explícita) y 0 en caso contrario. Los criterios de corrección se establecieron a partir de las respuestas de un grupo de 15 estudiantes universitarios obteniendo un acuerdo medio entre jueces de $96 \%$. En la segunda medida cada respuesta se puntuó con 1 (respuesta que incluía la idea principal), 0,5 (respuesta que incluía el tema pero no.la idea principal de la lectura), ó 0 (cualquier otra respuesta). La corrección fue realizada por el autor de la investigación y un colaborador que puntuaron las respuestas de forma independiente obteniendo un porcentaje de acuerdos de $88 \%, 92 \%$ y $83 \%$ en las pruebas de test, primer postest y segundo postest respectivamente. La muestra de jueces nos sirvió para confirmar que la idea principal era la que nosotros consideramos como tal, obteniendo un porcentaje medio de acuerdos de $91 \%$ entre las tres pruebas. La corrección de la tercera prueba se hizo dando 2 puntos cuando había coincidencia entre la respuesta correcta y la proporcionada por los niños, 1 punto cuando había una diferencia de un punto (p. e. debía valorar con 0 y valoraba con 1 ), y 0 puntos cuando había una diferencia de dos puntos. Los jueces universitarios puntuaron igualmente las frases para asegurarnos una correcta valoración, obteniendo un porcentaje medio de acuerdos de $82 \%$.

El segundo tipo de medidas se refirió a la conciencia de estructura textual, tomándose dos medidas en este caso. En la primera, adaptada de Richgels y col. (1987), se pidió a los niños que emparejaran diversos textos según la estructura organizativa que presentaran (comparativa o enumerativa), dando razones estructurales de dicho emparejamiento siendo los textos similares o los mismos que los utilizados en las pruebas anteriores. La medida se aplicó en el pretest y en el primer postest, obteniéndose un coeficiente alfa de 0.61. En la segunda medida se pedía a los niños que leyeran un texto durante 10 minutos y, tras una breve prueba distractora, se les daba una hoja en blanco para que escribieran durante otros 10 minutos y, tras una breve prueba distractora, se les daba una hoja en blanco para que escribieran durante otros 10 minutos todo aquello que pudieran recordar. Nuestra medida se refería al grado de correspondencia entre la estructura del recuerdo de los niños, y la estructura del texto previamente leído, clasificando dichos recuerdos en tres categorías siguiendo los criterios de (Brooks y Dansereau, 1983): a) organizado según la estructura del texto original; b) parcialmente organizado, y c) no organizado. Esta prueba fue aplicada en el primer y segundo postest, empleando dos textos diferentes de una longitud aproximada de 350 palabras y con estructura textual comparativa. Ambos textos tenían un contenido no incluido en el curriculum escolar.

La corrección de la primera medida de este grupo, emparejamiento de 
lecturas, se hizo otorgando 1 punto cuando los sujetos efectuaban un emparejamiento correcto y proporcionaban una razón claramente referida a la estructura, 0,5 puntos si había un emparejamiento correcto y se daba alguna razón aproximada referida a la estructura pero no formulada claramente, y 0 puntos en cualquier otro caso obteniendo un porcentaje de acuerdo .de $92 \%$ y $96 \%$ en las pruebas de test y postest respectivamente. Los porcentajes de acuerdo en la corrección de la segunda de las pruebas, fueron de $90 \%$ y $95 \%$ en el primer y segundo postest respectivamente.

Para medir el recuerdo de la información de textos previamente leídos, tercer tipo de medidas, presentamos a los niños los textos comparativos a los que acabamos de aludir en la segunda de las medidas anteriores en la forma allí explicada. Para la corrección, en ambos casos los textos fueron divididos en ideas-unidad siguiendo un procedimiento similar al expuesto por Mayer (1985). Estas ideas-unidad fueron clasificadas en tres niveles de importancia mediante un sistema de jueces, obteniendo un total de ideas de nivel 3 (muy importante) de 13 en primer postest y 12 en el segundo, de nivel 2 (bastante importante) 13 y 14 en cada caso, y de nivel 1 (poco importantes) 13 y 13 respectivamente. Las hojas de recuerdo de los niños fueron igualmente divididas en ideas-unidad, contabilizando a continuación cuántas ideas del texto estaban presentes en el recuerdo de los niños. Finalmente, tomamos tres medidas de recuerdo siguiendo a varios autores (Brown, Day y Jones, 1983; Armbruster, Anderson y Ostertag, 1987): a) recuerdo del tercio de ideas más importantes; b) recuerdo del tercio de ideas de mediana importancia, y c) recuerdo del tercio de ideas de menor importancia. La corrección fue efectuada por el autor de esta investigación y por un colaborador, el cual examinó un tercio de las contestaciones de los niños de los grupos experimental y control, obteniendo un porcentaje de acuerdo del $94 \%$ en el primer postest y $93 \%$ en el segundo, resolviendo las discrepancias de común acuerdo.

\section{RESULTADOS}

Los datos fueron analizados con un diseño de parcelas divididas (splitplot) (Edwards, 1979) mediante un análisis de varianza $2 \times 2$ con un factor entresujetos (tratamiento) y otro intrasujetos (tiempo) con medidas repetidas en todas las pruebas pretest-postest. En aquellas medidas en que no se aplicaron pruebas equivalentes antes y después del tratamiento los datos se analizaron de la forma que más adelante se explicará.

Los resultados del primer grupo de medidas, obtención de ideas principales de un texto, en el test y primer postest se pueden ver en la Tabla I. Los resultados del análisis de varianza no arrojaron efectos significativos para ninguno de los factores considerados aisladamente, excepto en el factor "tratamiento" de la prueba de valoración de oraciones $(\mathrm{F}(1,62)=5.5$, $\mathrm{p}<0.5)$. Sin embargo la interacción de ambos factores tratamiento $\times$ tiempo fue significativa en todos los casos, puntuando el grupo experimental más alto que el de control en el postest. Los resultados de dicha interacción fueron $F(1,62)=22.86, p<001$ para la primera prueba (idea principal explícita), $F(1,62)=7.60, p<.01$ para la segunda prueba (idea principal implícita), y $\mathrm{F}(1,62)=6,54, \mathrm{p}<.05$ para la tercera prueba (valoración de ora- 
ciones). En la misma Tabla se pueden ver los datos del segundo postest en el que, como se recordará, se perdieron algunos sujetos. Estos resultados fueron analizados con un análisis de varianza simple en el que se compararon los dos grupos de tratamiento, encontrándose diferencias igualmente significativas tanto en la primera de las medidas $(F(1,62)=10.958, \mathrm{p}<.01)$ como en la de captación de la idea principal implícita $(F(1,62)=5.813$, $\mathrm{p}<.05)$.

\section{TABLA I}

Media y (desviación típica) de las medidas dependientes de captación de ideas principales $(a: N=64 ; b: N=51)(T=$ test $; P-1=$ primer postest; $P-2=$ segundo postest)

\begin{tabular}{lcccccccc}
\hline & \multicolumn{3}{c}{$\begin{array}{c}\text { Idea principal } \\
\text { explicita }\end{array}$} & \multicolumn{3}{c}{$\begin{array}{c}\text { Idea principal } \\
\text { implícita }\end{array}$} & \multicolumn{3}{c}{$\begin{array}{c}\text { Valoración } \\
\text { de oraciones }\end{array}$} \\
\cline { 2 - 8 } & $\mathbf{T}^{\mathbf{2}}$ & $\mathbf{P}^{\mathbf{2}}$ & $\mathbf{P}^{\mathbf{b}}$ & $\mathbf{T}^{\mathbf{2}}$ & $\mathbf{P}^{\mathbf{2}} \mathbf{1}^{\mathbf{2}}$ & $\mathbf{P}^{\mathbf{b}}$ & $\mathbf{T}^{\mathbf{2}}$ & $\mathbf{P}^{\mathbf{2}}$ \\
\hline Grupo & 1.938 & 1.531 & 1.667 & 0.844 & 0.672 & 0.963 & 14.375 & 13.031 \\
Control & $(1.24)$ & $(1.11)$ & $(1.36)$ & $(0.72)$ & $(0.76)$ & $(0.83)$ & $(1.96)$ & $(4.02)$ \\
Grupo Expe- & 1.531 & 2.406 & 2.833 & 0.734 & 1.063 & 1.438 & 14.375 & 15.781 \\
rimental & $(1.19)$ & $(1.16)$ & $(1.13)$ & $(0.77)$ & $(0.74)$ & $(0.52)$ & $(3.20)$ & $(3.20)$ \\
\hline
\end{tabular}

Los resultados del segundo tipo de medidas referido a la conciencia de estructura textual se encuentran en las Tablas II y III. Por lo que respecta a la medida de emparejamiento de lecturas, el análisis de varianza $2 \times 2$ (tratamiento $\mathrm{x}$ tiempo) arrojó resultados significativos únicamente para la interacción de ambos factores al igual que en las medidas anteriores $(\mathrm{F}(1,62)=13.31, \mathrm{p}<.001)$, puntuando el grupo experimental más alto que el de control en el postest. Por lo que respecta a la segunda de las medidas de este grupo, grado de correspondencia entre la estructura del recuerdo de los niños y la estructura del texto previamente leído, los resultados fueron analizados con la prueba Chi-cuadrado encontrándose un índice de $4.302(p=0.116)$ para el primer postest y de $3.312(p=0.191)$ en el segundo, ambos no significativos, aunque los sujetos del grupo experimental tendieron a organizar su recuerdo utilizando una estructura comparativa en mayor grado que los del grupo de control.

\section{TABLA II}

Media y (desviación típica) de la medida de Emparejamiento de textos en el test y primer postest $(N=64 ; T=$ test $; P-1=$ primer postest $)$

\begin{tabular}{lcc}
\hline & $\mathrm{T}$ & $\mathrm{P}-1$ \\
\hline Grupo & 0.891 & 0.688 \\
Control & $(0.90)$ & $(0.77)$ \\
Grupo & 0.703 & 1.344 \\
Experimental & $(0.86)$ & $(0.75)$ \\
\hline
\end{tabular}


TABLA III

Número y porcentaje de niños con un recuerdo organizado según la estructura del texto leido en el primer postest $(N=64)$ y en el segundo postest $(N=51)$

\begin{tabular}{lcccccc}
\hline & \multicolumn{3}{c}{ Grado de correspondencia entre recuerdo y texto } \\
\cline { 2 - 7 } & \multicolumn{3}{c}{ Primer postest } & \multicolumn{3}{c}{ Segundo Postest } \\
\cline { 2 - 7 } & No organ. Parc. org. & Organ. & No organ. Parc. org. & Organ. \\
\hline Grupo & 22 & 5 & 5 & 5 & 8 & 14 \\
Control & $34 \%$ & $8 \%$ & $8 \%$ & $10 \%$ & $16 \%$ & $27 \%$ \\
Grupo Expe- & 15 & 5 & 12 & 1 & 6 & 17 \\
rimental & $23 \%$ & $8 \%$ & $19 \%$ & $2 \%$ & $12 \%$ & $33 \%$ \\
\hline
\end{tabular}

Los resultados acerca de recuerdo de las ideas de diferentes niveles, el último tipo de medidas dependientes, se pueden ver en la Tabla IV. Estos resultados fueron analizados con un análisis de varianza simple en el que se compararon los dos grupos de tratamiento, encontrándose diferencias significativas en el recuerdo del tercio de ideas más importantes pero únicamente en el segundo postest $(F(1,49)=4.662, p<.05)$, aunque en el primero de ellos hay que notar que las mayores diferencias se produjeron en el tercio de ideas más importantes no hallándose diferencias en el recuerdo de las ideas de menor importancia.

\section{TABLA IV}

Media y (desviación típica) de las medidas de recuerdo de ideas en el primer postest $(N=64) y$ en el segundo postest $(N=51)$

\begin{tabular}{lcccccc}
\hline & \multicolumn{4}{c}{ Recuerdo de ideas-unidad } \\
\cline { 2 - 7 } & \multicolumn{3}{c}{ Primer postest } & \multicolumn{3}{c}{ Segundo Postest } \\
\cline { 2 - 7 } & Niv. 1 & Niv. 2 & Niv. 3 & Niv. 1 & Niv. 2 & Niv. 3 \\
\hline Grupo & 1.094 & 1.344 & 2.031 & 3.815 & 5.963 & 4.593 \\
Control & $(1.67)$ & $(2.01)$ & $(2.63)$ & $(1.94)$ & $(2.98)$ & $(2.55)$ \\
Grupo Expe- & 1.938 & 1.531 & 2.415 & 5.167 & 6.125 & 5.458 \\
rimental & $(2.39)$ & $(2.17)$ & $(3.32)$ & $(2.20)$ & $(2.54)$ & $(2.17)$ \\
\hline
\end{tabular}

Niv. 1 = recuerdo del tercio más importante de ideas-unidad.

Niv. 2 = recuerdo del tercio de ideas-unidad de mediana importancia.

Niv. 3 = recuerdo del tercio menos importante de ideas-unidad.

\section{CONCLUSION}

El conjunto de resultados que acabamos de exponer demuestra la eficacia de nuestro programa de instrucción. Así, las hipótesis iniciales resultan básicamente confirmadas. Los niños del grupo experimental mejoraron la habilidad de captar la idea principal explícita e implícita de textos expositivos, y esta mejoría se mantuvo tras un período de cuatro meses. Igual- 
mente mejoró la habilidad de valorar la importancia relativa de las diversas frases de un texto según su nivel dentro de la estructura textual la cual suele ser deficitaria en niños de grados escolares medios (Winograd, 1984).

Los resultados referidos a la conciencia de estructura textual igualmente confirman nuestras previsiones. En la primera de las medidas, emparejamiento de textos con igual estructura, los sujetos experimentales puntuaron significativamente más alto que los de control como efecto del tratamiento aplicado. En la segunda medida, grado de correspondencia entre la organización del recuerdo de los sujetos y la estructura del texto, los resultados no llegan a ser significativos aunque hay una tendencia a organizar el recuerdo con una estructura equivalente a la del texto original en los niños que han seguido el programa instruccional. Se debe tener en cuenta que estos chicos no recibieron ningún tipo de entrenamiento específico en tareas de recuerdo, por lo cual los datos presentados, aunque no sean estadísticamente significativos, presentan una tendencia prometedora. La falta de concordancia entre ambas medidas de conciencia de estructura textual es coherente con otros estudios como el de Richgels y col. (1987) los cuales también encuentran resultados diferentes cuando emplean medidas de comprensión y de recuerdo para medir la conciencia de diversos tipos de estructuras textuales.

Por lo que respecta al tercer grupo de datos, los referidos al recuerdo de ideas del texto, nuestra cuarta hipótesis preveía que habría un efecto de generalización de los efectos del entrenamiento de forma que los sujetos del grupo experimental recordarian más ideas importantes que los del grupo experimental, no debiendo haber diferencias significativas en el recuerdo del resto de información. Los resultados confirman las previsiones, aunque únicamente encontramos diferencias significativas en el segundo postest. Este hecho creemos que puede explicarse sobre todo por el nivel de dificultad de los textos empleados en ambas pruebas. El texto utilizado en el primer postest («comparación entre las estaciones espaciales y transbordadores espaciales») tenía un contenido más difícil que el segundo («comparación entre los Juegos Olímpicos modernos y los de la antigua Grecia»), debido presumiblemente al menor desarrollo de los esquemas de conocimiento previo. Además, las condiciones de aplicación de esta prueba en el primer postest fueron peores que en el segundo, debido a limitaciones de tiempo que pudieron incidir en la fatiga de los niños: Todo esto creemos que puede contribuir a explicar no solamente la ausencia de diferencias significativas en la medida que estamos comentando en el primer postest, sino también las bajas puntuaciones de éste con respecto al segundo postest. No obstante los datos son prometedores y confirman básicamente los efectos de generalización que preveíamos a la vez que coinciden con los proporcionados en otros estudios (Meyer, Brandt y Bluth, 1980; Taylor y Samuels, 1983; Sánchez, 1988) y con las previsiones del modelo de van Dijk y Kintsch (1983), de los cuales se deduce que el seguimiento de la estrategia estructural por parte de los sujetos se debe traducir en un mayor recuerdo de la información, particularmente de aquélla que ocupa un lugar más importante en la macroestructura del texto.

La importancia de estos resultados se deriva de la consideración de dos tipos de datos. Por una parte, los macroprocesos lectores instruídos en nuestro estudio son la base de diversas técnicas de estudio, tales como el 
subrayado, la toma de notas, el resumen, la realización de esquemas o las técnicas para representar el contenido de los textos en forma de diagrama (Anderson y Armbruster, 1984). Por otra parte, hay diversos estudios que prueban cómo los profesores no prestan suficiente atención a estos macroprocesos en la enseñanza ordinaria (Durkin, 1978, 1979; Solé, 1987; Aarnoutse, 1989) lo que hace más necesaria una intervención al respecto.

\section{Referencias}

AARTNOUTSE, C. (1989). «Reading comprehension instruction: What is it and how to improve it?». Comunicación presentada a la Third European Conference for Research on Learning and Instruction. Madrid.

Anderson, T. H., y Armbruster, B. B. (1984). «Studying». En P. D. Pearson, Handbook of Reading Research. Nueva York, Longman.

ARMBRUSTER, B. B.; ANDERSON, T. H., y OSTERTAG, J. (1987). «Does text structure/summarization instruction facilitate learning from expository text?». Reading Research Quarterly, XXII, 3, 331-346.

BAKER, L., y BROWN, A. L. (1984). "Metacognitive Skills and Reading». En P. D. Pearson (ed.): Handbook of Reading Research. Nueva York, Longman.

BAUMANN, J. F. (1984). "The effectiveness of a direct instruction paradigm for training main idea comprehension". Reading Research Quarterly, $X X, 1$, pp. 93-115. (Traducción en Infancia y Aprendizaje, 31-32, 89-105).

BRitTon, B. K.; GlynN, S. M.; MEYeR, B. J. F., y PENLAND, M. J. (1982). «Effects of text structure on use of cognitive capacity during reading". Journal of Educational Psychology, $74,1,51-61$.

BrooKs, L. W., y DANSERAU, D. F. (1983). «Effects of structural schema training and Text Organization on Expsitory Prose Processing». Journal of Educational Psychology, 75, 6, $811-820$.

Brown, A. L.; DAY, J. D., y JONES, R. S. (1983). *The development of plans for summarizing texts". Child Development, 54, 968-979.

CAMPIONE, J. C., y ARMBRUSTER, B. B. (1985). «Acquiring information from texts: an analysis of four approaches». En J. W. Segal, S. F. Chipman, R. Glaser (eds.), Thinking and learning skills. (Vol. 1). Hillsdale, N. J., Lawrence Erlbaum Associates.

CIESI, H. L.; SPILICH, G. J., y VOSS, J. F. (1979). «Acquisiton of domain-related information in relation to high - and low- domain knowledge". Joumal of Verbal Learning and Verbal Bebavior, 18, 257-274.

COOK, L. K., y MAYER, R. E. (1988). "Teaching readers about structure of scientific text". Journal of Educational Psychology, 80, 488-456.

DANNER, F. W. (1976). "Children's understandings of intersentence organization in the recall of short decriptive passages». Journal of Educational Psychology, 68, 174-183.

DUFFY, G. G., y ROEHLER, L. R. (1982). "The illusion of instruction». Reading Research Quarterly, 17, 3, 438-445.

DURKIN, D. (1978-1979). *What classroom observations reveal about comprehension instruction». Reading Research Quarterly, 14, 481-533.

EDWARDS, A. L. (1979). Multiple Regression and the Analysis of Variance and covariance. San Francisco, Freeman.

ENGLERT, C. S., y HIEBERT, E. H. (1984). «Children's developing awareness of text structures in expository materialsm. Journal of Educational Psychology, 76, 1, 65-74.

ENGLERT, C.S., y THOMAS, C. Ch. (1987). "Sensitivity to text structure in reading and writing: a comparison between learning disabled and non-learning disabled students». Learning Disability Quarterly, 10, 93-105.

Garner, R.; Alexander, P.; Slater, W.; Hare, V. Ch.; SMITH, T., y Reis, R. (1986). “Children's knowledge of structural properties of expository text. Journal of Educational Pycho$\log y, 78,6,411-416$.

Glover, J. A.; Halpain, D. R.; DinNel, D. L.; MCKeE, T. K.; CORKILl, A. J., y WISE, S. L. (1988). «Effects of across-chapter signals on recall of text». Joumal of Educational Psycho$\log y, 80,1,3-15$.

HARE, V. Ch.; RABINowrtz, M., y SCHNiEBle, K. M. (1989). «Text effects on main idea comprehension». Reading Research Quarterly, XXIV, 1, 72-88.

KIERAS, D. E. (1979). Initial mention as a cue to the main idea and main item of a technical passage. Technical report núm. 3. University of Arizona.

- (1981). "Component processes in the comprehension of simple prose». Journal of Verbal Learning and Verbal Behavior, 20, 1-23. 
- (1982). «A model of reader strategy for abstracting main ideas from simple technical prosen. Text, 2, 47-82.

- (1985). *Thematic Processes in the Comprehension of Technical Prosen. En B. K. Britton y J. B. Black (eds.), Understanding Expository Text. Hillsdale, N. J., Lawrence Erlbaum Associates.

KINTSCH, W., y VAN DIJK, T.A. (1978). *Towards a model of discourse comprehension and production.. Psychological Review, 85, 363-394.

KINTSCH, W., y YARBROUGH, J. C. (1982). "Role of rethorical structure in prose comprehension. Journal of Educational Psychology, 74, 6, 828-834.

MAYER, R. E. (1985). «Structural Analysis of Science Prose: Can We Increase Problem-Solving Performance?". En B. K. Britton y J. B. Black (eds.), Understanding Expository Text. Hillsdale, N. J., Lawrence Erlbaum Associates.

MeYeR, B. J. F. (1985). «Prose Analysis: Purposes, Procedures, and Problems». En B. K. Britton y J. B. Black (eds.), Understanding Expository Text. Hillsdale, N. J., Lawrence Erlbaum Associates.

- (1987). "Following the author's top-level organization: an important skill for reading comprehension». En R. J. Tierney, P. L. Anders, J. N. Mitchell (eds.), Understanding readers' understanding, theory and practice. Hillsdale, N. J., Lawrence Erlbaum Associates.

MEYER, B. J. F.; BRANDT, D. M.,yBLUTH, G. J. (1980). *Use of top level structure in text: key for reading comprehension of ninth-grade students». Reading Research Quarterly, $X V I, 1,72-102$.

Meyer, B. J. F., y Freedle, R. O. (1984). "Effects of discourse type on recall», American Educational Research Journal, 21, 1, pp. 121-143.

NunNalLY, J. C. (1970). Introducion to Psychological Measurement. Nueva York: McGrawHill. (Traducción española, Introducción a la medición psicológica, Paidós, 1973).

OHLhausen, M. M., y RolleR, C. M. (1988). "The operation of text structure and content schemata in isolation and in interaction». Reading Research Quarterly, XXIII, 1, 70-88.

PALMERE, M.; BENTON, S. L.; Glover, J. A., y RONNING, R. R. (1983). «Elaboration and recall of main ideas in prose". Journal of Educational Psychology, 75, 6, 898-907.

Pearson, P. D.; Hansen, J., y GoRdon, C. (1979). "The effect of background knowledge on young children's comprehension of explicit and implicit information". Journal of Reading Behavior, 11, 201-209.

Pressley, M.; GoOdChild, F.; FleET, J.; ZajChOWSKI, R., y Evans, L. D. (1989). "The challenges of classroom strategy instruction». The Elementary School Journal, 89, 301-342.

RECHT, D. E., y LESLIE, L. (1988). «Effect of prior knowledge on good and poor readers' memory of text». Journal of Educational Psychology, 80, 1, 16-20.

RichGels, D. J.; MCGeE, L. M.; LomaX, R. G., y SHEARD, C. (1987). *Awareness of four text structures: Effects on recall of expository text». Reading Research Quarterly, $X X I I$, 2, $177-196$

RUBIN, A., y HANSEN, J. (1986). "Reading and Writing: How are the first two "R's" related?». En J. Orasanu (ed.), Reading Comprehension: from research to practice. Hillsdale, N. J., Lawrence Erlbaum Associates.

SÁNCHEZ, E. (1988). «Aprender a leer y leer para aprender: características del escolar con pobre capacidad de comprensión». Infancia y Aprendizaje, 44, 35-57.

SCARDAMALIA, M., y BEREITER, C. (1984). *Development of strategies in text processingm. En H. Mandl, N. L. Stein y T. Trabasso (eds.), Learning and comprehension of text. Hillsdale, N. J., Lawrence Erlbaum Associates.

SOLE, I. (1987). L'ensenyament de la comprensió lectora. Barcelona, Ceac.

STEVENS, R. J. (1988). «Effects of strategy training on the identification of the main idea of expository passages». Journal of Educational Psychology, 80, 1, 21-26.

TAYLOR, B. M., y BEACH, R. W. (1984). "The effects of text structure instruction on middle-grade students' comprehension and production of expository text". Reading Research Quarterly, XIX, 2, 134-146.

TAYLOR, B. M., y SAMUELS, S. J. (1983). "Children's use of text structure in the recall of expository material". American Educational Research Joumal, 40, 517-528.

VAN DIJK, T. A., y KINTSCH, W. (1983). Strategies of discourse comprebension. Nueva York. Academic Press.

VIDAL-ABARCA, E. (1989). «La comprensión lectora de ideas principales en textos expositivos del Ciclo Medio de la EGB: programa de instrucción y procesos cognitivos explicativos". Tesis Doctoral. Universidad de Valencia.

WILliams, J. P. (1984). «Categorization of macrostructure and finding main idea». Journal of Educational Psychology, 76, 874-879.

Williams, J. P.; TAYLOR, M. B., y GANGER, S. (1981). «Text variations at the level of the individual sentence and the comprehension of simple expository paragraphsm. Journal of Educational Psychology, 73, 851-865.

WINOGRAD, P. N. (1984). "Strategic difficulties in summarizing texts». Reading Research Quarterly, 19, 404-425. (Traducción española en Infancia y Aprendizaje, 31-32, 67-87).

YusSEN, S. R.; MATHEws, S. R., y HiEbeRT, E. (1982). «Metacognitive Aspects of Reading". En W. Otto y S. White (eds.). Reading Expository Material. Nueva York: Academic Press. 
A continuación se encuentra un ejemplo de un texto comparativo empleado en el programa de instrucción y la imagen gráfica que los niños habían de completar con las proposiciones del texto para representar la macroestructura textual.

«Los astros luminosos son aquellos que tienen luz propia, como por ejemplo el Sol. Por el contrario, los astros no luminosos son aquellos que no tienen luz propia, como la Tierra, Marte o la Luna.

Las estrellas son astros luminosos que podemos ver en el cielo durante las noches despejadas. Los planetas, satélites y cometas son astros no luminosos que también brillan en el cielo porque reflejan la luz de alguna estrella. Así, por ejemplo, la Luna brilla porque refleja la luz del Sol.

Mientras que las estrellas están muy alejadas unas de otras, los planetas y satélites se encuentran mucho más cercanos. Por ejemplo, la distancia entre La Tierra y el planeta Marte es mucho menor que la que hay entre el Sol y la estrella más cercana.

Otra diferencia entre los astros luminosos y los no luminosos es su tamaño. El Sol por ejemplo es mucho más grande que los planetas y satélites cercanos a él.

Por todo esto podemos decir que los astros tienen unas grandes diferencias con respecto a los astros no luminosos.»

A continuación se encuentra un ejemplo de texto con estructura enumerativa con la representación gráfica correspondiente.

"Cuando ocurre algo importante, como puede ser un suceso político o deportivo que interesa a gran cantidad de personas, los medios de comunicación lo dan a conocer. Hay diversos tipos de medios de comunicación.

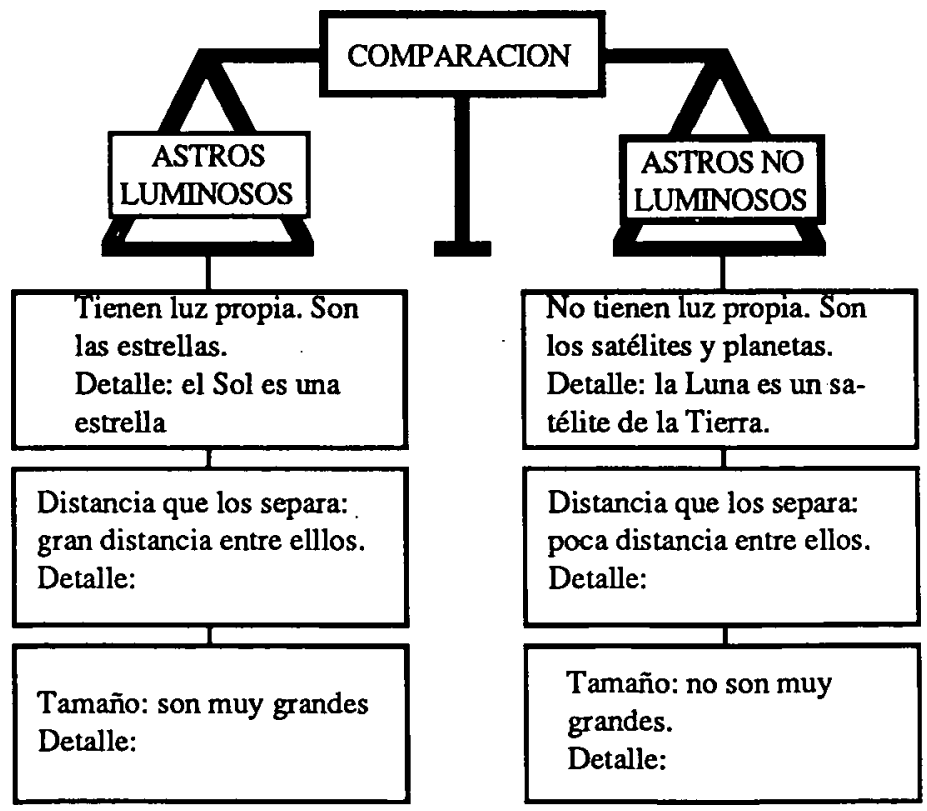


La prensa es un medio de comunicación. Es el medio de comunicación más antiguo de todos. La prensa está formada por los periódicos y las revistas, los cuales transmiten información a través del lenguaje escrito. La prensa se publica diaria, semanal y quincenalmente.

Otro medio de comunicación es la radio, que transmite la información a través de la palabra hablada. Hay distintas emisoras de radio como la Cadena SER, Radio Popular o Radio Nacional de España, en las que podemos escuchar programas informativos, musicales, etc.

Un tercer medio de comunicación importante es la televisión. La televisión es el medio de comunicación más reciente. Utiliza la palabra hablada y la imagen, lo que nos permite escuchar una información al mismo tiempo que pode- mos ver imágenes representativas de las cosas que han ocurrido. La televisión es el medio de comunicación que más se utiliza en la actualidad.»

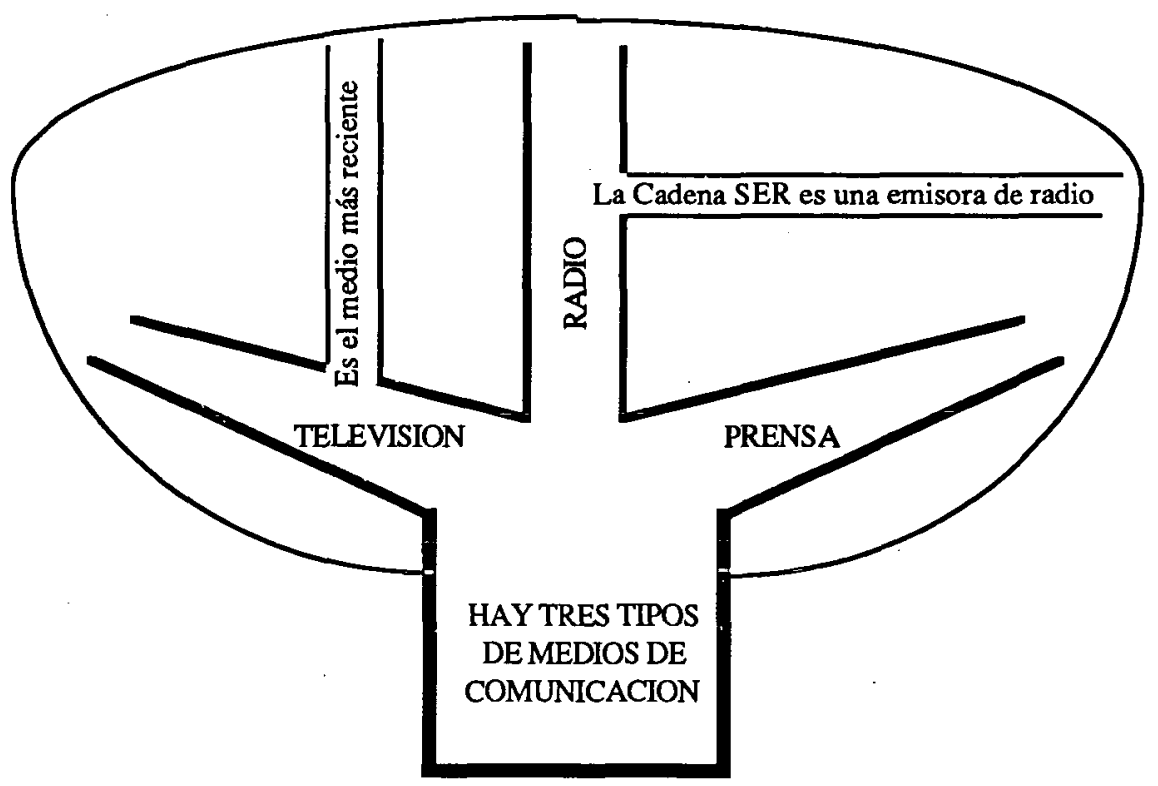

\section{Extended Summary}

The skill of getting main ideas from texts is studied within the framework of thematic processes, and it involves macroprocesses of text structure formation. The comprehension model on which the present work is based, is the strategic model proposed by van Dijk and Kintsch. According to this model, the reader applies several macrorules and macrostructures to the text he/she has read. Thus, macroprocesses involved in creating a macrostructure of the text imply the interaction of two kinds of variables, i.e., those which are related to the written text, and those related to the reader. A particular aspect of this interaction, and one to which this reaserch has paid special attention, is the textual structure. An awareness of this structure, together with the use of B. J. F. Meyers' «structural stra- 
tegy", have been shown to be relevant in capturing main ideas, and in recalling and ranking ideas in a written text.

Three hypotheses on the direct effects of the instructional programme were formulated. It was expected that this programme would improve: 1) the skill of grasping the main idea, either explicit or implicit, in an expository text; 2) the skill of ranking ideas in a text according to their relative importance in the text structure; 3 ) awareness of enumerative and comparative text structures. Also, it was expected that these positive effects would be maintained in the follow-up study several months later. The instructional programme covered each of these skills, however, some generalization to other skills was also predicted. A fourth hypothesis was added, i.e., predicting that there would be an improvement in the experimental group's skill for recalling the most important information in written texts.

Two groups of fifth grade children attending different schools in Valencia served as experimental and control group. The instructional programme was carried out at one of these schools, during school hours over a period of six weeks for twelve sessions of $\mathbf{4 5}$ minutes each. A direct instruction model was used. Children were taught to use a structural strategy to distinguish the different levels of importance of propositions.

Three types of measures were taken. The first, referred to understanding the main idea in a text, and was tested through the three following tasks: a) writing down the main idea, explicity stated, in four different texts; b) writing down the implicit main idea in two different texts; and, c) evaluating five sentences from two of the test passages according to their relative importance to the textual structure. Two equivalent versions of each of these tasks were administered to the study and control groups at the beginning and end of the instruction programme. A second postest was administered four months later, after two revision sessions, only on the first two tasks.

The second type of measures, assessed the subject's awareness of a textual structure. The two following tasks were used to test this skill: a) matching two texts according to their textual structure and explaining the reasons for the match; b) children were asked to read a text with a comparative structure, they then had to carry our a task aimed at distracting them; later they were asked to write down everything they could recall from the text. The degree of correspondence between the text structure and the one recalled by subject's was scored. Recall was classified as "organized" if it had the same comparative structure as the text; "partially organized" or «not organized» were the two other alternatives. Two equivalent versions of the first task on textual awareness were used at the pre- and postest. The second task was used only on the first and second postest.

The third type of measure, tested the skill to recall the most important information in the text. The recall task used was the same as the one described in the previous paragraph. However, here three partial measures were taken: 1) the recall of one third of the most important content ideas; 2) the recall of one third of the content ideas of average importance;3) the recall of one third of the least important content ideas or details. This task was carried out in the first and second postests.

The data was analyzed through a split-plot design, carrying out an 
ANOVA with two factors on all the pre- and postest measures. The first factor, i.e., treatment, was related to the differences between the experimental and control group; the second, was a time factor (pre- and postest) with repeated measures. Results, from the first types of measures applied, did not show significant differences for any of the individual factors except in the case of the treatment factor on the third dependant measure. Nevertheless, as predicted, treatment time was significant in all cases; the experimental group scored higher than the controls on both postests. In the follow-up study these improvements were maintained. In relation to the second study measures, this effect was also found on the text matching task used to measure textual structure awareness. However, test results from the second task were not significantly different, though a higher percentage of the recall of children in the experimental group was classified as «organized". With respect to the third dependant measure, recall of one third of content ideas of greater importance was found to differ significantly in favour of the experimental group, however, only in the second postest.

These results show the effectiveness of the instructional programme. Initial hypotheses were, overall, confirmed. This study is important for a number of reasons; first, the macroprocesses taught in this study are the basis for a number of study techniques; and, secondly, research studies have show that teachers do not pay enough attention to these processes in daily instruction. 